Mens

revue d'histoire intellectuelle de l'Amérique française

\title{
Zacharie Vincent : dernier Huron et premier artiste autochtone de tradition occidentale
}

\section{Louise Vigneault}

Volume 6, numéro 2, printemps 2006

URI : https://id.erudit.org/iderudit/1024304ar

DOI : https://doi.org/10.7202/1024304ar

Aller au sommaire du numéro

Éditeur(s)

Centre de recherche en civilisation canadienne-française

ISSN

1492-8647 (imprimé)

1927-9299 (numérique)

Découvrir la revue

Citer cet article

Vigneault, L. (2006). Zacharie Vincent : dernier Huron et premier artiste autochtone de tradition occidentale. Mens, 6(2), 239-261.

https://doi.org/10.7202/1024304ar
Résumé de l'article

Cet article porte sur la démarche et l'œuvre de l'artiste huron Zacharie Vincent qui, au cours du XIX ${ }^{\mathrm{e}}$ siècle, s'est consacré à la peinture de la tradition occidentale académique. Il a produit un nombre considérable d'autoportraits et de scènes de la vie quotidienne, ce qui lui a permis de renverser les schèmes de représentation en vigueur du Sujet autochtone et de répondre au discours alarmiste à l'égard du sort de sa communauté. Cet essai constitue le premier jet d'une étude visant à cerner les stratégies de représentation et de diffusion des œuvres de cet artiste qui a réussi à témoigner de sa réalité et de celle de sa communauté auprès de l'intelligentsia et de l'élite politique canadienne et britannique. 


\title{
ZACHARIE VINCENT : DERNIER HURON ET \\ PREMIER ARTISTE AUTOCHTONE DE TRADITION OCCIDENTALE
}

\author{
Louise Vigneault \\ Département d'histoire de l'art \\ et d'études cinématographiques \\ Université de Montréal
}

\section{Résumé}

Cet article porte sur la démarche et l'œuvre de l'artiste huron Zacharie Vincent qui, au cours du $\mathrm{XIX}^{\mathrm{e}}$ siècle, s'est consacré à la peinture de la tradition occidentale académique. Il a produit un nombre considérable d'autoportraits et de scènes de la vie quotidienne, ce qui lui a permis de renverser les schèmes de représentation en vigueur du Sujet autochtone et de répondre au discours alarmiste à l'égard du sort de sa communauté. Cet essai constitue le premier jet d'une étude visant à cerner les stratégies de représentation et de diffusion des œuvres de cet artiste qui a réussi à témoigner de sa réalité et de celle de sa communauté auprès de l'intelligentsia et de l'élite politique canadienne et britannique.

\section{Abstract}

This article presents the artistic process and works of Huron artist Zacharie Vincent who; throughout the 19th century, was dedicated to Western academic painting. He produced many self-portraits and scenes of daily life, thus changing bow the Aboriginal was represented and responding to an alarmist discourse on the future of his community. This paper is the first in a study of the representation and exbibition strategies of an artist who succeeded in presenting bis reality and that of his community to the Canadian and British intelligentsia and political elite. 
Zacharie Vincent a été, au cours du XIX ${ }^{\mathrm{e}}$ siècle, le premier membre de la communauté huronne à entreprendre une carrière de peintre, suivant les paramètres de la tradition académique occidentale. Plutôt méconnu de nos jours, cet artiste a pourtant réussi, de son vivant, à attirer l'attention d'un public important constitué de l'élite politique canadienne et britannique. Bien que certains historiens, anthropologues et historiens de l'art se sont intéressés à l'homme et à son œuvre', leurs recherches sont demeurées fragmentaires. En tirant bénéfice de ces précieux résultats, nous avons entrepris de confronter les données relatives au contexte de production des œuvres et à la situation de la communauté huronne de Lorette (près de Québec) de l'époque aux récentes réflexions sur les stratégies d'affirmation culturelle et identitaire des communautés minoritaires ${ }^{2}$. Cet essai présente donc les résultats préliminaires d'une étude visant à mieux comprendre le discours de représentation de cet artiste qui est passé du rôle passif de modèle d'un portrait réalisé en 1838 par Antoine Plamondon, à celui résolument actif d'auteur d'autoportraits et d'illustrations de scènes quotidiennes témoignant de la réalité de l'artiste et des siens. Nous verrons ainsi de quelles manières Vincent a réussi à imposer une image renouvelée de la réalité huronne du $\mathrm{XIX}^{\mathrm{e}}$ siècle, à l'aide de tactiques d'appropriation des éléments de culture de l'Autre et de langages de représentation ambivalents.

\section{Portrait du « dernier Huron »}

À la fin des années 1980, l'historien de l'art FrançoisMarc Gagnon ${ }^{3}$ publiait une analyse du portrait en pied de Zacharie Vincent intitulé Le dernier des Hurons (huile sur toile) réalisé en 1838 par Plamondon. Il y cernait alors une identification symbolique à la communauté huronne chez de nombreux Canadiens français, en raison de leur sort commun. Dans 
le contexte des années qui suivaient la défaite des Patriotes, le Sujet autochtone était alors considéré comme un symbole de perte d'autonomie et de renoncement de ses fondements culturels et politiques face au pouvoir étranger. Le succès que récoltait l'œuvre, tout comme la popularité du thème de l'indianité en général auprès des Canadiens français, s'expliquerait, selon Gagnon, par l'engouement pour le thème de la disparition de la race, au lendemain de la Conquête et de la défaite des Patriotes de 1837-1838.

Afin de souligner la victoire de Plamondon au concours de la Société littéraire et historique de Québec, un article anonyme était publié le 30 avril 1838 dans le journal Le Canadien $^{4}$. On y trouvait une description de Vincent suivant les stéréotypes de l'indianité en vigueur, mais également une allusion littérale à la métaphore entre le sort des Autochtones et celui des Canadien français :

Il [Plamondon] nous a représenté son sauvage, debout dans une attitude imposante, guerrière et méditative, les bras croisés sur la poitrine, le front levé vers le ciel ; il l'a paré au milieu de ses bois, auxquels il semble dire un dernier et solennel adieu, pour lui et toute sa race; en un mot il a vraiment peint le dernier des Hurons. Lorsqu'on fixe pour la première fois ses longs cheveux noirs, bouclés et flottant sur ses épaules, ses traits éminemment caractéristiques, son teint cuivré, ses yeux étincellants [sic], sa belle draperie de couverte, sa ceinture à laquelle est suspendue [sic] son coutelas, on reconnait bien le fils des hommes libres, le chasseur et le guerrier des vastes forêts, le canoteur des grands lacs, le dernier rejeton d'une nation noble et intrépide, qui a disparu devant nous comme le castor de nos rivières, les élans de nos bois ; et comme nous-mêmes, peutêtre, nous disparaitront devant une nation plus puissante. Le fort chasse le faible [...] Puissions-nous élever 
quelques monuments de nous-mêmes avant d'être engloutis dans le flot de l'émigration ${ }^{5}$ !

Selon Gagnon, Plamondon mettait moins en scène une simple représentation du dernier Huron qu'une allégorie des enjeux de la survivance de la communauté canadienne-française. En projetant sur l'Autochtone ses propres inquiétudes, et en utilisant ce dernier comme énonciateur, il détournait toutefois sa parole réelle, comme le remarque l'auteur :

Le dernier des Hurons n'est pas un portrait ethnographique. C'est un tableau symbolique où le personnage de Zacharie Vincent prête son corps, son attitude et son destin à l'expression d'un sentiment de résignation sans doute partagé par plusieurs des compatriotes de Plamondon ${ }^{6}$.

L'artiste faisait appel, en fait, au langage rhétorique de la prosopopée visuelle qui consiste à projeter ses propres sentiments sur un élément externe, une personne absente, à s'approprier son discours et à le faire sien. Au lendemain de la rébellion des Patriotes qui faisait naître chez les Canadiens français de vives inquiétudes quant à leur avenir, Plamondon se montrait donc sensible aux menaces de disparition de la nation huronne, d'autant plus qu'il était natif de l'Ancienne Lorette, et avait sans doute une certaine connaissance, même minime, de la situation et de l'histoire de la communauté autochtone adjacente. Toutefois, en raison de son allégeance à l'Empire britannique, et donc de son penchant pour l'ordre et la sécurité au détriment de la résistance pouvant engendrer l'anarchie, Gagnon rappelait que l'artiste faisait adopter à son modèle une attitude de "résignation contemplative ${ }^{7}$ ».

Ce portrait de Vincent, tout comme la popularité du thème de l'indianité, inspirait peu après l'historien FrançoisXavier Garneau pour son poème intitulé «Le dernier des Hurons ». Les allégeances nationalistes de ce dernier lui ins- 
piraient toutefois une finale teintée d'espoir, où les minorités dépossédées se voyaient éventuellement vengées, comme le révèle cet extrait :

Qui sait peut-être alors renaîtront sur ces rives

L'indien et ses sombres forêts

Mes ayeux laisseront leurs ombres fugitives

Qui n'ont ni culte ni paix,

Et se levant comme après un long rêve

Ils verront partout les mêmes lieux,

Les sapins descendants jusqu'aux flots sur la grève

En haut les mêmes cieux ${ }^{8}$.

Si nous appuyons la lecture de Gagnon, nous croyons qu'elle mérite d'être complétée par certaines données mettant en lumière le sort de la communauté huronne elle-même, sa possible répercussion chez les Canadiens français ainsi que la manière dont Vincent aurait réagi et rétorqué à cet état de fait.

En 1836, soit peu avant la réalisation de l'œuvre de Plamondon, un rapport sur les Affaires indiennes concluait que l'importance du métissage, chez la population huronne, remettait en question l'intégrité de leur identité : «By the Intermixture of White Blood they have now so lost the original Purity of Race that they cannot properly be considered as Indians. ' " Conscients de l'enrichissement que peut constituer certains emprunts, dans un contexte d'échanges interculturels volontaires, et ne partageant pas les valeurs occidentales d'intégrité ethnique, les membres de la communauté huronne ne niaient pas cet état de fait, comme ils l'expliquaient clairement une dizaine d'années après la publication de ce rapport:

[...] comment pourrions-nous ne pas aimer les blancs? Notre race est maintenant mêlée à la leurs [sic]. Nous avons épousé des femmes blanches et les hommes blancs ont épousé des Sauvagesses. Plus des trois quarts 
du village sont habités par les [gens] issus de ces Mariages entre mêlés et à peine $\mathrm{y}-\mathrm{a}-\mathrm{t}-\mathrm{il}$ un sauvage de pur sang dans tout le village ${ }^{10}$.

Face au destin de ceux qui avaient été jadis leurs alliés dans le cadre des campagnes de traite et des conflits avec les Iroquois, les Canadiens français manifestaient une ardente empathie à leur égard, mais éprouvaient du même coup une cuisante inquiétude quant à leur propre avenir politique. Cette appréhension sera d'ailleurs ravivée peu de temps après, alors que lord Durham arrivait à Québec, le 27 mai 1838, à titre de nouveau gouverneur. Sensible à l'œuvre de Plamondon, Durham faisait une offre d'achat pour le portrait de Vincent qu'il rapportait même en Angleterre, quelques mois plus tard ${ }^{11}$. Pour l'élite britannique, le Sujet autochtone constituait une curiosité prisée, un modèle qui permettait d'enrichir la galerie des héros romantiques en vogue. Si Plamondon et ses collègues ont pu interpréter ce geste du gouverneur comme une marque de sympathie à l'égard de la population francophone, ils déchantèrent rapidement lorsque l'année suivante, ce dernier présentait son célèbre Report on the Affairs of British North America (1839) dans lequel il promouvait l'assimilation des Canadiens français.

Quant au principal intéressé, Vincent lui-même, tout porte à croire qu'il a assumé volontiers cette auréole légendaire de "dernier Huron ", en prenant soin toutefois d'en trafiquer le sens et le langage. À ce titre, on le voit apparaître l'année suivante dans une cuvre du peintre anglais Henry D. Thielcke intitulée Nomination du chef Robert Symes, le 21 février 1838 (figure 1) ${ }^{12}$. Symes, dit Hosa8athi qui signifie "il a défendu son pays ", était un citoyen de Québec d'origine anglaise. Il s'était démarqué grâce à certains exploits significatifs, comme celui de prêter main-forte aux victimes, lors de l'épidémie de choléra en 1834 , et pour avoir occupé la fonc- 


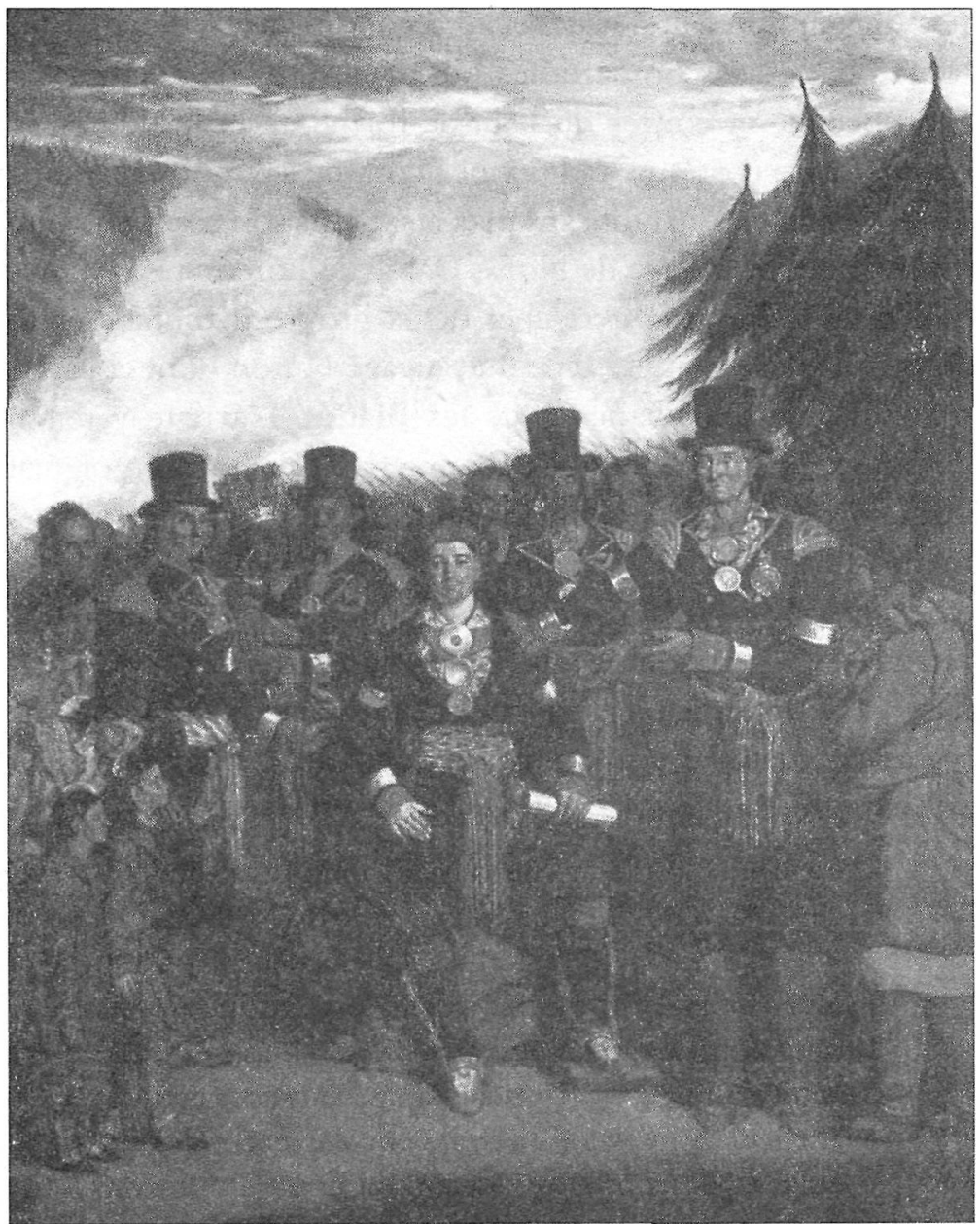

Figure 1 : Henry D. Thielcke, Nomination du chef Robert Symes, le 21 février 1838,1839, huile sur toile, $150 \mathrm{~cm}$ x $127 \mathrm{~cm}$. Collection du Château Ramezay. 
tion de magistrat, dans le district de Québec. L'artiste a choisi, par conséquent, de le représenter en trône et entouré de l'élite des chefs de la nation huronne. Parmi ces dignitaires, Zacharie Vincent apparaît à gauche, au second plan. Il se démarque toutefois de ses compatriotes qui sont vêtus de costumes à l'européenne et coiffés du haut-de-forme en peau de castor, en portant le costume traditionnel, comme le rapportait l'abbé Lindsay, plus de quarante ans plus tard, dans son compte rendu de l'histoire du village de Notre-Dame de Lorette : «Le jeune indien coiffé d'un couvre-chef de sa propre manufacture figure au tableau comme type du sauvage Huron dont la lignée n'a subi aucune alliance avec les Blancs. L'artiste y tenait comme souvenir historique de la race. ${ }^{13}{ }^{\prime}$ Ce choix témoignait donc d'une volonté, chez Vincent, de se démarquer clairement des autres représentants de la nation, mais également des figures héroïques autochtones que l'histoire officielle a retenues, en fonction de leur intégration aux valeurs occidentales et de leur action positive à l'endroit de la communauté blanche, comme ces personnages dont l'abbé Lindsay relatait la mémoire :

C'est Totiri, capitaine huron, du bourg de Saint-Joseph, qui embrassa un des premiers le christianisme et convertit la moitié de sa cabane en chapelle. [...] C'est Joseph Chihaten8a, dont la sainteté fut si manifeste que les missionnaires l'invoquèrent après sa mort. C'est Téondéchoren, c'est Tsondatsaa, qui, adonnés au vice avant leur conversion, deviennent des exemples de probité et de constance. Mais celui dont le nom est resté le plus célèbre, et dont la mémoire est encore vivante dans la tribu, c'est Ahasistari, le brave d'entre les braves. Miraculeusement préservé de maint danger $[\ldots]$ avant que la "bonne nouvelle » eût retenti à ses oreilles, cet homme remarquable avait eu comme une 
intuition du Vrai Dieu que les pères venaient lui faire connaître et aimer ${ }^{14}$.

Ces Autochtones consentants, prêchant pour la cause du christianisme, ou répondant à l'image héroïque du martyr, servaient ainsi directement le discours dominant ${ }^{15}$. Au contraire de ces exemples, Vincent exposait plutôt une image de résistance, aussi bien dans son attitude que dans les attributs utilisés.

\section{Quelques données biographiques et historiques}

On sait peu de chose sur Vincent, si ce n'est qu'il était âgé de 23 ans lorsqu'il a posé pour Plamondon, et qu'il a entrepris à son tour, par la suite, de se consacrer à la peinture, sous le tutorat de ce dernier qui lui a donné quelques conseils ${ }^{16}$. Développant un style où l'approche académique côtoie la fraîcheur de quelques maladresses techniques, il a produit une série d'autoportraits et de scènes de la vie quotidienne - plus de six cents dessins et peintures ${ }^{17}$ - qui traduisent sa vision de la situation contemporaine de la communauté huronne. Ces œuvres deviendront en fait un moyen efficace de diffuser cette vision et surtout, de répliquer au ton nostalgique, voire alarmiste, de l'œuvre de son maître. Avant de fournir une description de son œuvre, prenons d'abord connaissance de quelques données biographiques qui apporteront quelques éclaircissements sur le contexte de production.

Zacharie Vincent (1815-1886) dit Telari-o-lin, qui signifie "sans mélange, non divisé ", est né au village Huron de Lorette, où il a passé la majeure partie de sa vie et a occupé la fonction de chef $^{18}$. Son père, Gabriel Vincent, était reconnu pour avoir cherché à éduquer ses enfants suivant la tradition ancestrale, comme le rappelle cette notice nécrologique publiée dans le Quebec Star le 8 avril 1829 : 
DIED - On Friday, 29th ult., at Indian Lorette, near this city, 8en8adahronhé, or Gabriel Vincent, third chief of the Hurons residing at that village. He was the only remaining Indian of the village who had descended in a direct line, without intermixture of blood, from the original tribe inhabiting the borders of Lake Huron. He was also one who retained most of the habits, and the only one who reared his family in the use of the language of his forefathers, the younger inhabitants of the village now speaking the French language only and not understanding their own ${ }^{19}$.

Malgré cette initiative, le fils, Zacharie, s'est intéressé à certaines pratiques culturelles de tradition occidentale et a occupé diverses fonctions comme celle de dépisteur pour la garnison britannique. Marie Falardeau, une iroquoise qu'il épouse en 1848, lui donnera quatre enfants : Cyprien né en 1848, Gabriel en 1850, Zacharie en 1852 et Marie en 1854. L'aîné, Cyprien, sera toutefois le seul, à notre connaissance, à figurer dans les portraits de son père. Par le portrait familial intitulé Zacharie Vincent et son fils Cyprien (non daté, huile sur toile), Vincent offrait une réponse directe à l'œuvre de Plamondon : il présentait en fait une preuve tangible de la survivance de sa nation, et ce, malgré les menaces séculaires de son anéantissement et le constat de métissage.

Il est d'ailleurs symptomatique de constater que la traduction de son nom huron, "sans mélange, non divisé ", a été récemment l'objet d'une révision. Les auteurs du Dictionnaire biographique du Canada affirmaient que «la présence de deux noms européens, Bergevin du côté paternel, et Otis (Hôtesse, ou "celui qui passe au-dessus des faîtes des maisons") du côté maternel, laisse croire que ni Gabriel Vincent ni son fils n'étaient de sang $\operatorname{pur}^{20} »$. Le sociologue Léon Gérin avait expliqué, au sujet des appellations européennes, que depuis le $\mathrm{XVII}^{\mathrm{e}}$ siècle, les Hurons avaient remplacé progressivement 
les noms exclusifs partagés par les membres de mêmes clans par les noms de famille distincts et transmissibles de père en fils. À leur arrivée en Huronie, les missionnaires avaient attribué aux nouveaux baptisés des noms de saints - Vincent, Bastien (Sébastien), Romain, Gonzague, etc. - lesquels restaient toutefois jumelés aux noms claniques et transmis aux générations suivantes. Vers la fin du XVIII ${ }^{e}$ siècle, ces noms ont fini par remplacer les anciennes nominations claniques. Ils étaient utilisés comme noms propres et étaient transmis systématiquement de père en fils ${ }^{21}$. Marie-Dominique Labelle notait, plus récemment, que de récentes études réalisées en onomastique amérindienne et en linguistique suggèrent que l'appellation "Telari-o-lin » dériverait plutôt du terme mohawks "Tekarioken » qui signifie " parole qui se divise en deux en sortant de la bouche", évoquant ainsi une identité métissée sous-jacente, ou encore une attitude ambivalente, dualiste $^{22}$. Cette nouvelle interprétation serait plausible, si on considère que son épouse, Marie Falardeau, était Iroquoise, et aurait pu de cette manière enrichir l'appellation d'un sens nouveau. Labelle souligne du même coup la parenté linguistique et les liens rompus entre Iroquoiens et les WendatsIroquoiens du Saint-Laurent, depuis l'exode ${ }^{23}$. Cette question demeure toutefois en suspens.

Avant d'exposer la démarche artistique de Vincent, rappelons brièvement les événements qui ont provoqué le processus d'acculturation et de métissage des Hurons de Lorette. Au moment de l'arrivée de Cartier, les Hurons (ou Wyandots) occupaient la région du sud de l'Ontario et avaient une réputation enviable parmi les nations autochtones. Semi-sédentaires, ils formaient des villages qu'ils habitaient entre dix et quinze ans, suivant des unités sociales matrilinéaires. À l'arrivée de Champlain, vers 1608 , de nombreux villages avaient déjà disparu. Au cours de la décennie 1630, la population 
s'est trouvée décimée aux deux tiers, en raison des maladies infectieuses transmises par les missionnaires et des conflits avec les Iroquois. Selon l'historien Alain Beaulieu, on a évalué la population huronne de la décennie 1640 à environ 9000 individus seulement ${ }^{24}$. Peu après 1650 , les survivants se dispersaient. Une fraction de la population trouvait refuge en territoire autochtone aux États-Unis, une autre dans le comté ontarien d'Essex, tandis qu'une troisième se réfugiait dans un territoire, la Jeune ou la Nouvelle Lorette (Wendake), situé au nord-ouest de Québec ${ }^{25}$. Les Britanniques ont reconnu cette concession, en vertu du «Traité » de Murray $(1760)^{26}$. En 1762, le gouverneur n'évaluait plus cette population huronne qu'à une centaine ${ }^{27}$. Malgré ce constat alarmiste, la communauté se montrait très active sur le plan commercial, en se consacrant notamment au troc du maïs, et en reprenant ses habitudes de chasse ${ }^{28}$. Comme par le passé, elle se trouvait sollicitée pour la traite des fourrures. Lorsque ce commerce connut un déclin, au début du $\mathrm{XIX}^{\mathrm{e}}$ siècle, la redéfinition des intérêts qui liaient traditionnellement les Premières Nations aux Britanniques devint inévitable. Désormais marginalisés par les enjeux politiques et économiques du nouveau pays, les $\mathrm{Hu}$ rons se sont tournés vers la fabrication et le commerce d'objets exotiques ou dits touristiques - raquettes, souliers mous, couvertures, mocassins, paniers, etc. - afin d'assurer leur subsistance. Certains de ces objets, destinés à un commerce florissant, étaient même fabriqués sur une base industrielle. Comme le résume Beaulieu, "Entre 1650 et 1760, le mode de vie des Hurons de Lorette va connaitre des modifications importantes. Tous les observateurs s'entendent pour dire qu'il s'agit de la communauté dont les manières s'apparentent le plus à celles des Canadiens $^{29}$ ». La présence des peuples européens avait ainsi ébranlé la nation huronne dans ses fonde- 
ments aussi bien démographiques qu'économiques, politiques et culturels.

\section{Pratiques artisanales : acculturation et métissage}

Les scènes de la vie quotidienne réalisées par Vincent témoignent d'ailleurs de cette acculturation et du métissage culturel. Si les personnages s'adonnent à des activités tirées de la tradition autochtone - la fabrication de canots, de raquettes, l'incurvation du bois, le tissage des cadres, etc. - ils prennent place toutefois dans un décor occidental. L'artiste prend même soin de rapporter les détails du mobilier: une chaise cannée à la canadienne, un sofa de style victorien avec son accoudoir à col de cygne, une table à piétement de bois tourné, une horloge octogonale aux chiffres romains, un cadre ovale renfermant la photographie en buste d'une femme aux cheveux noirs bouclés... Quant aux artisans aux cheveux sombres et courts, ils portent des vêtements canadiens : une veste de style "édouardien " sur une tunique blanche, des pantalons et des chaussures de cuir ${ }^{30}$. Seules les activités auxquelles ils se livrent permettent leur identification aux Premières Nations. Il est significatif, à cet égard, de constater que la majorité des scènes représentent des personnages consacrés à des activités artisanales. Cette réalité renvoie, non seulement à la transmission de pratiques ancestrales, à la mémoire séculaire de la communauté, mais également à ce qui est devenu pour ses descendants un mode de vie substitutif, voire palliatif. Si ces scènes intérieures décrivent une réalité métissée, les scènes extérieures - comme Scène de campement et Fabrication de canot (non datées, lavis et encre sur papier) - décrivent des pratiques qui évoquent, pour le spectateur occidental, une réalité autochtone révolue, figée dans un présent ethnographique. Ainsi, l'artiste aurait-il cherché à souligner ici le fait que ces activités étaient désormais réduites à 
des mises en scène stéréotypées réservées à l'unique regard touristique?

S'il est hasardeux de prêter certaines intentions à l'artiste ou d'interpréter ses œuvres, compte tenu de la rareté des données, il est toutefois possible, à la lumière des informations concernant l'identité symbolique de l'artiste, au langage stylistique et iconographique des œuvres, ainsi qu'au contexte sociopolitique de l'époque, de détecter certaines stratégies d'affirmation culturelle et identitaire. Puisque l'identité est par définition un élément instable, variable, qui se redéfinit sans cesse à partir de la manière dont un individu ou un groupe se perçoit et dont il est perçu, dans le cas d'une communauté minoritaire, confrontée à un contexte contraignant qui n'offre pas l'espace adéquat pour imposer sa représentation, cette identité peut s'exprimer tantôt sous forme de manque, tantôt sous forme de renversement ou d'hybridation des codes dominants. Ces tactiques permettent d'échapper efficacement aux catégorisations, à un essentialisme ethnique et aux stéréotypes en vigueur, et d'imposer une représentation ajustée de la situation contemporaine d'un individu et de son groupe d'appartenance.

Dans son essai consacré au colonialisme culturel et aux catégorisations sémantiques dont les institutions muséales font usages dans leurs fonctions taxonomiques, James Clifford expliquait qu'un des problèmes majeurs contribuant à maintenir les objets culturels et artistiques des communautés dites périphériques dans une position de marginalisation et d'infériorité est la notion de "préservation culturelle». Celle-ci consiste à considérer ces objets comme les résultats d'une pratique anonyme et prescriptive, issue d'une tradition séculaire et, par conséquent, hors de toute temporalite ${ }^{31}$. Dans cette optique, les œuvres qui ne répondent pas aux critères artistiques occidentaux fondés sur les valeurs d'authenticité, d'ori- 
ginalité, d'homogénéité et de pureté, se trouvent automatiquement exclues du mainstream culturel. Le maintien de ces critères entraîne une marginalisation des nombreuses pratiques hybrides. Entrent notamment dans cette catégorie les objets touristiques, soi-disant " faux », qui présentent des signes de " contamination » de la culture occidentale ${ }^{32}$. Cet état de fait expliquerait le recours de certains artistes autochtones à des stratégies telles que la récupération de moyens de diffusion occidentaux ou l'affirmation de traits résultant de métissage culturel. Ces tactiques permettent alors aux cultures minoritaires et marginalisées d'assurer leur autonomie et de conserver un certain contrôle de leurs biens culturels ${ }^{33}$.

Dans son étude consacrée aux stratégies politiques de résistance des communautés minoritaires, Richard Handler a souligné que, lorsqu'un peuple est forcé d'abandonner son mode de vie qui assurait jusqu'à ce jour son autonomie afin d'adopter le commerce touristique, la fabrication d'objets dits " exotiques ", sa vie quotidienne devient une suite de performances et d'autoreprésentations qui ont pour effet d'objectiver et de trivialiser sa réalité sociale ${ }^{34}$. À ce titre, l'initiative de Vincent de s'approprier le médium pictural européen peut être interprétée comme une riposte à cet état de fait, mais également un moyen d'illustrer la réalité huronne dans toute sa complexité, avec ses emprunts, ses influences, ses pertes et ses acquis. En récupérant à son profit l'identité du " peintre de chevalet ", véritable archétype de l'artiste européen, Vincent s'assurait en fait une visibilité au sein de l'élite politique, comme le témoignait un article anonyme du quotidien The Star en 1887: «He painted pictures of himself and sold them to Lord Durham, Lord Elgin, Lord Monk, the Princess Louise and others. ${ }^{35}$ " En assurant la diffusion de son œuvre dans l'espace même de la culture hégémonique, l'artiste était en mesure du même coup de transmettre une image complexe, 
et donc plus complète, de l'Autochtone contemporain, et ce, malgré toutes les contradictions qu'elle renferme.

\section{Du portrait à l'autoreprésentation}

Dans l'autoportrait intitulé Zacharie Vincent dit Teliarolin (figure 2), l'artiste se représente en buste et confronte directement le spectateur. Le cadrage se resserre sur ce personnage rayonnant, flamboyant, qui occupe pratiquement tout l'espace. Le paysage forestier de l'arrière-plan, perceptible de chaque côté du visage, présente non pas un horizon sombre et crépusculaire, comme c'était le cas dans le portrait de Plamondon, mais un ciel dominé par des couleurs chaudes. Vincent est revêtu, pour sa part, de riches ornements : brassards, bracelets, pendants d'oreilles, tomahawk-pipe, couvre-chef d'argent à plumes, gorgerin (médaillon d'argent porté au cou), wampum. Sa tunique est entourée d'une ceinture fléchée, tandis que sur son torse trône une médaille de paix à l'effigie de la reine Victoria, de celles que recevaient à l'époque les chefs. L'abbé Lindsay rappelle, à ce sujet que

Jusqu'en 1854, on distribuait annuellement à la plupart des familles du village de Lorette des articles divers qu'on désignait sous le nom de " présents du Roi » [...] qui consistaient en munitions de chasse (poudre, balles, plomb, etc.) en couvertures de laine, drap bleu, indienne, couteaux, chaudières de cuivre, etc. On donnait aussi aux chefs, après leur élection, et de la part du souverain régnant, une riche et belle médaille en argent, frappée à l'effigie du roi ou de la reine d'Angleterre $^{36}$.

Vincent tient finalement dans sa main gauche (ou droite, si l'artiste a utilisé un miroir) le mât d'un drapeau dont il est possible de reconnaître un pan aux couleurs britanniques. Ce 


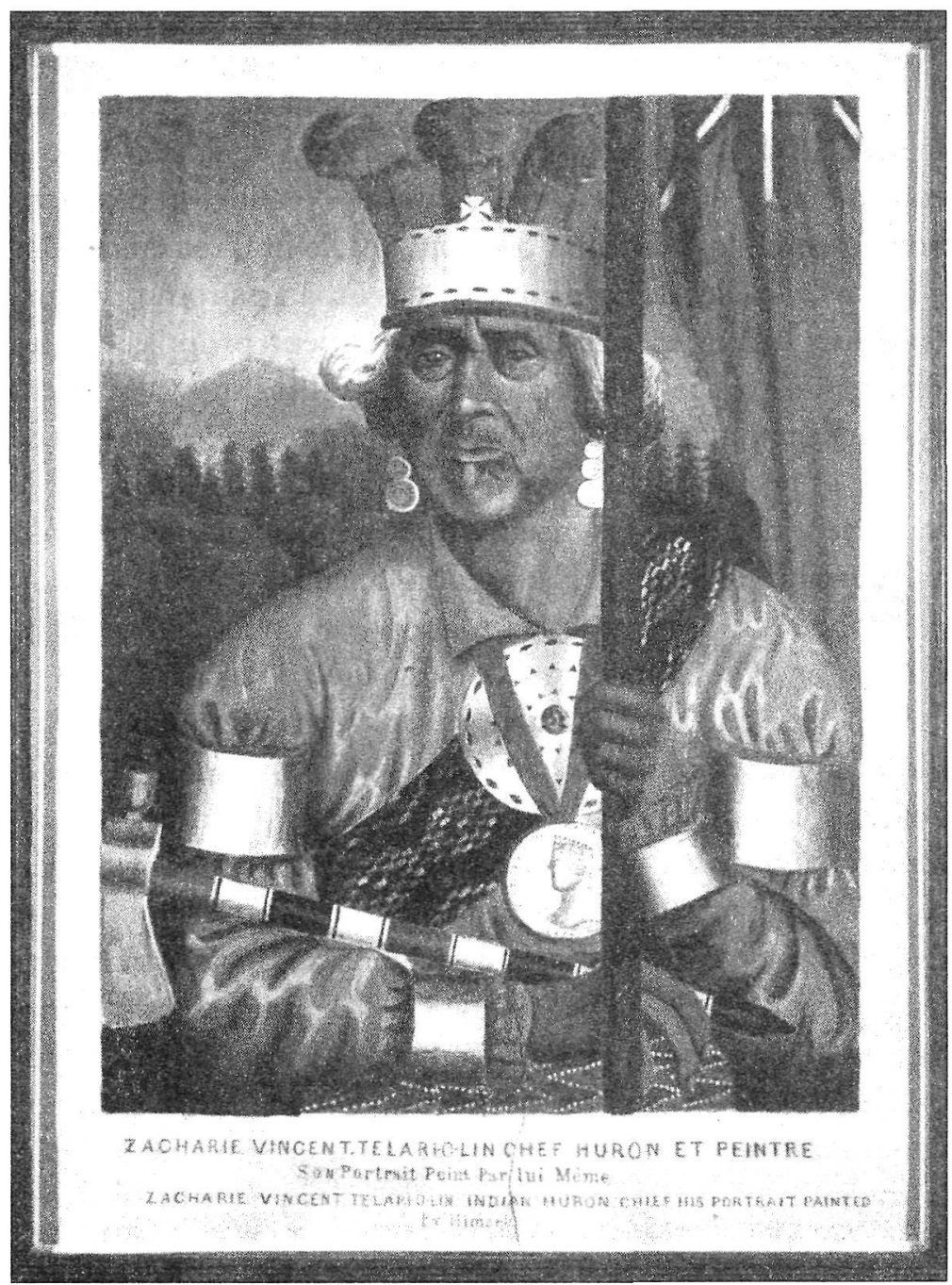

Figure 2: Zacharie Vincent dit Teliarolin, [Autoportrait], non daté, huile sur papier, $92,7 \mathrm{~cm} \times 70,8 \mathrm{~cm}$. Collection du Château Ramezay. 
détail évoque sans doute la fonction de dépisteur qu'aurait occupée l'artiste.

Les nombreux autoportraits, qui présentent en fait de simples variantes du premier, rassemblent sensiblement les mêmes attributs décoratifs témoins à la fois de la survie de la communauté et de son acculturation. Certains de ces attributs, comme les médailles de traite, résultent directement des alliances entre les deux peuples ou des échanges commerciaux. Face aux armes et aux ustensiles que leur proposaient les Blancs, dès les premiers contacts, les Autochtones auraient volontairement modifié plusieurs techniques de chasse, de guerre et de travail ${ }^{37}$. Par cette multiplication de détails, et le traitement de l'œuvre, Vincent offrait visiblement une réponse au portrait que réalisait Plamondon quelques années plus tôt. Au jeune Huron fier et résigné, il opposait un monarque flamboyant, en pose d'apparat. Cette initiative pourrait également être lue comme une tentative de création d'une image synthèse de la communauté, véritable figure synecdotique de la population huronne qui contrevenait radicalement aux représentations romantiques ou stéréotypées dominantes aussi bien sur la scène artistique que dans l'imagerie populaire. L'examen des autoportraits révèle également un détail symptomatique : les œuvres sont généralement dépourvues de signature et de datation et portent l'unique inscription de "Telario-lin ». Volontaire ou non, l'omission de signature suscite certaines interrogations concernant le statut de son auteur. Dans la tradition picturale prémoderne, le sujet peignant ('’artiste) tend à s'effacer devant le sujet peint. Seule la signature, qui vient momentanément contrecarrer l'effet d'illusion de la surface, rend justice à son auteur. Or, chez Vincent, le Sujet peignant s'efface volontiers derrière et le Sujet peint, lequel s'avère ici le même et qui, en occupant tout l'espace de repré- 
sentation, tend à incarner efficacement cette image réinterprétée de l'indianité.

\section{Hybridité et ambivalence}

En utilisant un moyen d'expression occidental pour représenter sa communauté en processus d'acculturation, Vincent détournait ainsi à son profit les valeurs, les paramètres et les privilèges de la culture dominante. Il contrevenait par le fait même au discours d'assimilation "résignée » véhiculée par Plamondon. Il mettait en place une tactique d'absorption et de mutation des codes de l'intérieur même du système imposé par l'Autre, plutôt que de s'opposer directement à ceuxci. Bien entendu, on ne saurait nier le fait que la présence d'éléments métissés ainsi que l'emprunt à un mode d'expression associé à la majorité présentent des signes de l'assimilation des Hurons, depuis plusieurs générations. En juxtaposant toutefois des éléments issus de la culture occidentale au matériel culturel autochtone, Vincent évitait que ses œuvres soient considérées par l'élite comme des objets commerciaux parmi tant d'autres ou comme de simples curiosités. Ainsi, l'artiste ne refoulait pas la réalité du métissage culturel, affirmant au contraire son existence pour mieux la transgresser, tout comme la proie imite son prédateur, de façon à le duper et à échapper ainsi à son emprise. En utilisant une image métissée, celle-là même que refusait la culture majoritaire au profit des représentations romantiques, Vincent brouillait ainsi les pistes. Michel de Certeau rappelait à cet effet que si le Sujet issu d'un groupe dominant tend à s'approprier le matériel culturel de l'Autre, afin d'enrichir la sienne, les Sujets appartenant à un groupe marginalisé emploieront autrement les valeurs et produits imposés par l'ordre dominant, plutôt que de les rejeter ${ }^{38}$. Le sociologue rapportait, à titre d'exemple, la manière dont certains groupes d'Autochtones utilisaient 
des éléments et des rituels espagnols (produits, lois, règles), en les subvertissant en les utilisant à d'autres fins que celles qui leur avaient été prescrites. De la même manière, Vincent a choisi d'intégrer les normes de la culture canadienne-française en y imposant les éléments de sa propre réalité. À ce titre, il réalisait une double transgression: celle de s'infiltrer dans le réseau artistique, par l'utilisation d'une forme de représentation occidentale, afin de créer la confusion quant à ses intentions réelles, et celle d'imposer à la culture majoritaire une image ambiguë de l'Autochtone.

Devant la menace d'une éventuelle disparition du peuple huron, Vincent se serait ainsi inscrit à la fois dans la réalité contemporaine autochtone et dans celle de la culture dominante qui le faisait exister dans cet espace conquis. À ce sujet, de Certeau rappelait que les minorités se trouvent régulièrement confrontées à un "non-lieu " duquel il devient impossible de distinguer leur propre espace de celui de l'Autre, ce qui justifie l'utilisation - volontaire ou involontaire - de tactiques de simulation, de résistance, d'astuce et de bricolage de la réalité. L'impossibilité de pouvoir se soustraire réellement et définitivement à cet espace comme à ces codes culturels justifierait également la résurgence de ce type d'action ${ }^{39}$. À ce sujet, Homi Bhaba parle pour sa part de l'« espace divisé de l'énonciation" (split-space of enunciation), qui permet d'introduire une conceptualisation de la culture basée non pas sur l'identité exotique ou fantasmée, mais sur celle d'une hybridité culturelle assumée ${ }^{40}$. Face à ce constat, une question émerge : et si la véritable identité de Vincent et des siens résidait justement dans cette ambiguité, cet entre-deux, à la frontière même du clivage culturel ? 


\section{NOTES}

${ }^{1}$ Anne-Marie Sioui, «Zacharie Vincent : un œuvre engagé ? ", Recherches amérindiennes au Québec, vol. XI, $\mathrm{n}^{\circ}$ 4, 1981, pp. 335-337 ; Marie-Dominique Labelle et Sylvie Thivierge, «Un peintre huron du XIX siècle : Zacharie Vincent ", Recherches amérindiennes au Québec, vol. XI, no 4 (1981), pp. 325-333; François-Marc Gagnon, "Antoine Plamondon, Le demier des Hurons (1838) 》, The Journal of Canadian Art History / Annales d'bistoire de l'art canadien, vol. 12, no 1 (1989), pp. 68-79.

${ }^{2}$ Nous remercions la direction et la gestion des archives du Château Ramezay pour leur collaboration.

${ }^{3}$ Gagnon, «Antoine Plamondon », pp. 68-79.

${ }^{4} I b i d$., p. 69.

${ }^{5}$ Ibid., p. 70.

${ }^{6}$ Ibid., pp. $72-73$.

${ }^{7}$ Ibid., p. 72 .

${ }^{8}$ Le Canadien, le 12 août 1840. Rapporté par Gagnon, «Antoine Plamondon », p. 70.

${ }^{9}$ " Report of a Committee of the Executive Council, [...] of the $7^{\text {th }}$ October 1836, respecting the Indian Department ", dans Copies or Extracts of Correspondance Since 1st April 1835, between the Secretary of State for the Colonies and the Governors of the British North American Provinces respecting the Indians in those Provinces, The House of Commons, 1839, p. 32. Rapporté par Alain Beaulieu, "Les Hurons de Lorette, le "traité de Murray" et la liberté de commerce" (1995), dans Denis Vaugeois, dir., Les Hurons de Lorette, Québec, Septentrion, 1996, pp. 269-270.

${ }^{10}$ Les « Hurons de Lorette à son Excellence le très Honorable Charles Theophilus Baron Metcalf, Lorette, 18 octobre 1845 ", Archives nationales du Canada, série RG10, bob. C-11481, vol. 122, pp. 5678-5679. Rapporté dans Beaulieu, «Les Hurons de Lorette... », p. 270.

${ }^{11}$ Gagnon, «Antoine Plamondon », p. 68 ; Claude Thibault, Trésors des communautés religieuses de la ville de Québec, Québec, Musée du Québec, 1973, p. 94.

${ }^{12}$ Lionel Lindsay, Notre-Dame de la jeune Lorette en la Nouvelle-France : étude bistorique, Montréal, Cie de publication de la Revue canadienne, 1900, p. 240.

${ }^{13}$ Ibid., pp. 272-273. 
${ }^{14}$ Ibid., pp. 266-267.

${ }^{15}$ Bertrand Gervais, "Éléments pour une rhétorique de l'assimilation », Recherches amérindiennes au Québec, vol. XVII, $\mathrm{n}^{\circ} 3$ (1987), p. 45.

${ }^{16}$ Labelle et Thivierge, « Un peintre huron du XIX ${ }^{\mathrm{e}}$ siècle », p. 326.

${ }^{17}$ David Karel, Marie-Dominique Labelle et Sylvie Thivierge, « Vincent, Zacharie ", Dictionnaire biographique du Canada, Sainte-Foy, Les Presses de l'Université Laval, [1966-2001], version électronique : http://www.biographi.ca/ FR/ShowBioPrintable.asp?BioId=40005 (consulté le 7 mars 2006).

${ }^{18}$ Ibid.

${ }^{19}$ Lindsay, Notre-Dame de la jeune Lorette, p. 269.

${ }^{20}$ Karel, Labelle et Thivierge, «Vincent, Zacharie ».

${ }^{21}$ Léon Gérin, « Le Huron de Lorette » (1901-1902), dans Vaugeois, dir., Les Hurons de Lorette, p. 48.

${ }^{22}$ Marie-Dominique Labelle, compte rendu manuscrit, archives du Château Ramezay. Cette confusion linguistique renvoie sans doute au fait que les deux termes renferment la notion de division, laquelle peut avoir pris un sens négatif ou positif, selon le contexte.

${ }^{23}$ Denys Delâge et Cornelius Jaenen ont appuyé cette thèse, tandis que Bruce Trigger, Claude Chapdelaine et Marcel Trudel l'ont refutée. Jacques Rousseau et George W. Brown rapportaient, dans le Dictionnaire biographique du Canada, que « De nos jours, on croit que les Hurons et les Iroquois ont eu une origine commune dans les régions du Sud ; ils seraient venus vers les Grands Lacs en suivant la ligne de l'Ohio ; ils se seraient séparés sur les bords des lacs Érié et Ontario, certains se dirigeant du côté nord de ces lacs, d'autres vers le sud. » ("Les Indiens du Nord-Est de l'Amérique », vol. 1 , Sainte-Foy, Les Presses de l'Université Laval, 1982, p. 7).

${ }^{24}$ Beaulieu, « Les Hurons de Lorette... », p. 262.

${ }^{25}$ Voir notamment Gérin, «Le Huron de Lorette » (1901-1902), Christian Morissonneau, «Développement et population de la réserve indienne du village-huron, Loretteville » (1970), Denys Delâge, «Les Hurons de Lorette dans leur contexte historique en 1760 ", dans Vaugeois, dir., Les Hurons de Lorette, pp. 21-41, 81-94, 97-131 ; Bruce Trigger, Les enfants d'Aataentsic, l'bistoire du peuple buron, traduit par Jean-Paul Sainte-Marie et Brigitte Chabert Hacikyan, Montréal, Libre Expression, 1991 ; Georges E. Sioui, Les Wendats : une civilisation méconnue, Sainte-Foy, Presses de l'Université Laval, 1994. 
${ }^{26}$ Voir Marcel Trudel, «Les Hurons et Murray en 1760 », et Beaulieu, «Les Hurons de Lorette... ", dans Vaugeois, dir., Les Hurons de Lorette, pp. 133-159, 255-295.

${ }^{27}$ Beaulieu, « Les Hurons de Lorette... », pp. 269-270.

${ }^{28}$ Trudel, « Les Hurons et Murray en $1760 »$, p. 144.

${ }^{29}$ Beaulieu, «Les Hurons de Lorette... », pp. 266-267.

${ }^{30}$ Fiches techniques, archives du Château Ramezay. Tiré de Donald B. Webster, The Book of Canadian Antiques, Toronto, McGraw-Hill Ryerson, 1974.

${ }^{31}$ James Clifford, " On Collecting art and Culture », Russel Furguson, Martha Gever, Trinht Minh-Ha, et al., Out There: Marginalization and Contemporary Culture, Cambridge, MIT Press, 1990, pp. 141-143.

${ }^{32}$ Ibid., pp. 146-147.

${ }^{33}$ Ibid., p. 149.

${ }^{34}$ Richard Handler, Nationalism and the Politics of Culture in Quebec, Madison, University of Wisconsin Press, 1988, p. 80.

${ }^{35}$ The Star, novembre 1887. Rapporté par Sioui, Les Wendats, p. 335.

${ }^{36}$ Lindsay, Notre-Dame de la jeune Lorette, pp. 273-274.

${ }^{37}$ Rousseau et Brown, «Les Indiens du Nord-Est de l'Amérique », p. 9.

${ }^{38}$ Michel de Certeau, Arts de faire, Paris, 10/18, 1980, pp. 11-12.

${ }^{39}$ Ibid., pp. 9-29.

${ }^{40}$ Homi Bhaba, "The Commitment to Theory ", New Formations, $\mathrm{n}^{\circ} 5$ (été 1988), p. 22. 